\title{
Tribological behaviour of multilayered textile composites: The effect of reciprocating sliding frequency
}

\author{
M.T. Mathew ${ }^{\mathrm{a}}$, Naveen V. Padaki ${ }^{\mathrm{b}}$, R. Alagirusamy ${ }^{\mathrm{b}}$, B.L. Deopura ${ }^{\mathrm{b}}$, R. Fangueiro $^{\mathrm{c}}$, \\ L.A. Rocha ${ }^{\mathrm{a}}$, J.R. Gomes ${ }^{\mathrm{a}, *}$ \\ a Centre for Mechanical and Materials Technologies (CT2M) and Department of Mechanical Engineering, University of Minho, Azurém, 4800-058 Guimarães, Portugal \\ b Department of Textile Technology, Indian Institute of Technology-Delhi, Hauz Khas, New Delhi 110016, India \\ ${ }^{\mathrm{c}}$ Department of Textile Engineering, University of Minho, Azurém, 4800-058 Guimarães, Portugal
}

\section{A R T I C L E I N F O}

\section{Article history:}

Received 5 September 2008

Received in revised form 5 January 2009

Accepted 5 January 2009

\section{Keywords:}

Multilayer textile composites

Frequency

Wear mechanisms

Reciprocating sliding

\begin{abstract}
A B S T R A C T
Textile composites have been used for various applications because of their enhanced strength/weight ratio and versatile properties compared to other materials such as metals. Many studies have investigated the tribological behaviour of textile composites, but none have focused on the tribological characterization of 3D multilayered woven reinforced textile composites. Five types of 3-ply woven interlocked structures with varying interlacements were used as reinforcement for the nylon fibre/polyester resin composites for the present study. The influence of the textile structure interlacement on the tribological properties of the composite material (in terms of wear volume) was investigated in this work. Further, special attention was given to understand the effect of sliding frequency on the tribological behaviour and driving wear mechanisms. The tests were conducted on a new class of reciprocating sliding wear tester, in dry (unlubricated) conditions, under a fixed applied load of $20 \mathrm{~N}$ by varying the frequency of oscillating motion $(0.5 \leq f \leq 8 \mathrm{~Hz})$. In addition, tests were also conducted, at a constant frequency of $4 \mathrm{~Hz}$ and as a function of several loads ( $5 \mathrm{~N}$ to $40 \mathrm{~N}$ ). From these tests, a 3-ply woven reinforced composite with the best tribological performance as a function of frequency and load was identified. It was observed that the type of woven structure had an influence on the tribological properties. Therefore, the selection of a textile composite should be based on the load and frequency at the service condition. The wear mechanisms involved in the tribological process were also analysed.
\end{abstract}

(c) 2009 Elsevier B.V. All rights reserved.

\section{Introduction}

Textile reinforced composites are a major type of composite materials used widely for aerospace, naval, defence, automobile and civil applications. Fibre reinforced composites are used not only for their structural properties, but also for electrical, thermal, tribological, chemical and environmental purposes for a variety of industrial applications [1]. It is important to characterize the tribological behaviour of such composites as they are employed in a broad a range of applications where friction and wear phenomena are also involved.

During last few years, several studies have reported the tribological behaviour of textile polymer composites [2-13]. Significant progress was made by Nak-Ho and Suh [2] and Lee et al. [3] on the friction and wear behaviour of textile polymer composites as a function of varying fibre orientations with respect to the sliding direction. The former observed for graphite fibre-epoxy composites

\footnotetext{
* Corresponding author.

E-mail address: jgomes@dem.uminho.pt (J.R. Gomes).
}

that both wear and friction coefficients were low when the orientation of the fibres was normal to the sliding surface. In Kevlar-epoxy composites when the fibres were oriented normal to the surface and the sliding direction, the wear volume loss was also low but the friction coefficient was the highest [3]. Shangguan and Cheng [4] studied the tribological properties of lanthanum treated carbon fibres reinforced PTFE composite under dry sliding conditions as a function of frequency. In detailed study of the effect of fibre orientation on the wear behaviour of carbon-polyether ether ketone (PEEK) and Kevlar-epoxy laminates [5], it was shown that the wear rate did not significantly change for fibre orientation variations of up to $30^{\circ}$ from the normal orientation. An important influential factor was whether the abrasive material compressed the fibre or tended to pull the fibre out from the composite as it passed. The latter mechanism of fibre pull-out generated a higher wear rate.

In an interesting study of glass fibre reinforced poly(vinyl)butyral-modified phenolic composites, comparison between wear performance of woven roving, plain weave and satin weave fabric reinforcement geometries has been carried out. It was observed that the fabric geometry had a significant influence on the friction and wear behaviour of the composites [6]. The wear rate was low- 


\begin{tabular}{|c|c|}
\hline \multicolumn{2}{|c|}{ Nomenclature } \\
\hline$c$ & contact field \\
\hline$i$ & interlacing field \\
\hline$f$ & float field \\
\hline$I$ & Interlacement Index \\
\hline$i_{\mathrm{wp}}$ & interlacements in warp \\
\hline & interlacements in weft \\
\hline \multicolumn{2}{|c|}{$R_{1}$ and $R_{2}$ warp and weft repeat of woven design } \\
\hline$V_{\mathrm{f}}$ & fibre volume fraction of the composite \\
\hline & weight of fabric $\left(\mathrm{g} / \mathrm{m}^{2}\right)$ \\
\hline & weight of composite $\left(\mathrm{g} / \mathrm{m}^{2}\right)$ \\
\hline \multicolumn{2}{|c|}{$\begin{array}{l}\text { N3P1, N3P2, N3P3, N3P4, N3P5 The 3-ply multilayer fabric } \\
\text { samples }\end{array}$} \\
\hline \multicolumn{2}{|c|}{ Greek letters } \\
\hline & composite density \\
\hline$\sigma_{\mathrm{f}}$ & fibre density \\
\hline
\end{tabular}

est for the plain weave glass fabric composite, while the woven roving composite exhibited greater tensile, flexural, impact and interlaminar shear strengths. Yoshioka and Seferis [7] performed tension fatigue tests of resin transfer moulded woven carbon fabric composites. They recommended a model to predict the modulus deterioration under tensile fatigue damage conditions based on a combination of the crimp model and the shear-lag model. Tsang et al. [8] reported fatigue properties of vibration-welded nylon 6 and nylon 66 reinforced with glass fibres. In general, low weld pressure appeared to give better fatigue performance than high weld pressure in vibration-welded nylons. In the absence of hysteretic heating, fatigue cracks initiated in the weld material. Fibre debonding and matrix micro-cracking lead to fatigue crack propagation and fast fracture under cyclic loading [9]. Recently, the same authors of the current study [10] have conducted an elaborative study of the tribological behaviour of textile warp-knit woven polymer composites as a function of applied load and sliding distance in dry and lubricated contact conditions. Textile polymer composites with different orientations and resins were considered for dry and lubricated contact conditions. It was found that a composite biaxial warp-knit reinforced with epoxy resin was the most suitable for tribological applications. Further, many other interesting works were reported on the tribological process of the textile composites and acting wear mechanisms [11-13].

Most textile composites have been made by stacking layers of woven preforms leading to prominent delamination failure under performance/application conditions [14]. Multilayer interlocked fabrics are a specific class of preforms, which can also fulfil the requirements for damage resistant composites, but have been scantily explored to achieve interlocking of fabric layers during the weaving stage [15-17]. The manufacturing of preforms of interlocking fabrics is advantageous because they are cost effective and the manufacturer has control over layer interlocking density based on weave variations apart from imparting higher impact and delamination resistance to the fibre reinforced composites. So, an attempt was made to study the effect of a multilayer structure on the sliding wear behaviour by developing five different multilayer interlocked woven structures with varying interlacements for composite reinforcement applications.

There are no specific studies reported, till today, on the tribological response of textile composites as a function of sliding frequencies. However, from the practical point of view, such aspects are very significant as the composites are exposed to varying sliding frequencies when used, for example, for ship structure and aerospace applications. This study investigated the effect of sliding frequency

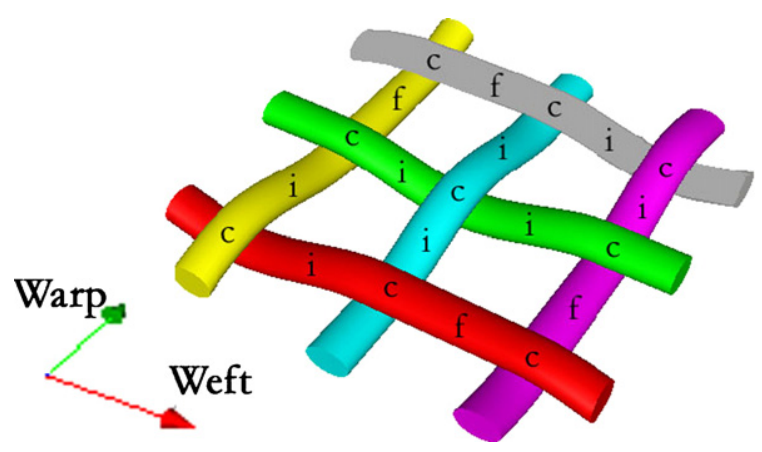

Fig. 1. Fabric fields.

on the tribological behaviour of multilayered textile composites using a new class of reciprocating sliding tribometer in dry (unlubricated) conditions. The main objectives were to understand the dominant wear mechanisms as they related to the oscillating motion and frequency for dry sliding conditions and to determine which of five composites had the best tribological behaviour.

\section{Materials and methods}

\subsection{Textile polymer composites: woven preforms and} Interlacement Index (I)

Woven preforms have two sets of yarns (warp and weft, Fig. 1) perpendicular to each other that are interlaced by a weaving process. Division of woven fabric area to three types of fields has been suggested by Selivanov, in a Russian journal, reviewed and reported by Milasius et al. [18]. The field types are defined as contact (c), interlacing (i) and float fields (f) (Fig. 1). A contact field is defined as the projected region occupied by both warp and weft thread systems. An interlacement field is the region between two contact fields where there is cross-over of warp yarn from one plane to another because of weaving around a weft yarn, and vice versa. When the yarn between two contact fields does not shift from one plane to another it is termed as float field.

One study generalized the structure-property correlation of the woven structure using an integrated factor based on interlacements [19]. The factor is known as the Interlacement Index $(I)$ and is defined by Eq. (1). The Interlacement Index is the ratio of the number of interlacement fields in any given weave repeat to the maximum possible number of contact fields in the design, where $i_{\mathrm{wp}}$ and $i_{\mathrm{wf}}$ are interlacements in warp and weft, respectively. The product of warp and weft repeat $\left(R_{1} \times R_{2}\right)$ of a woven design is equal to the maximum possible number of contact fields in the woven design repeat. The highest interlacement is seen for plain woven structures $(I=2)$ and non-interlaced structures would have an $I$ value of zero.

$I=\left(\frac{i_{\mathrm{wp}}+i_{\mathrm{wf}}}{R_{1} R_{2}}\right)$

The influence of structural variations on the wear behaviour of composites has been investigated for plain and satin structures [20], but investigations of the tribological properties of multilayered textile composites with respect to structural factors such as the Interlacement Index have not yet been performed.

\subsection{Multilayer woven preforms}

The 3-ply multilayer fabric samples were woven on a four harness flexible rapier automatic loom (Dornier), at $400 \mathrm{rpm}$ with 24 ends/cm and 12 picks/cm settings. Five meter lengths of five varieties of nylon 3-ply fabrics (N3P1-5) were woven using high 

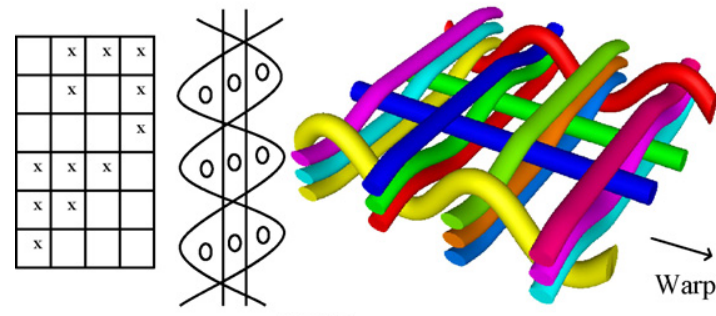

N3P1
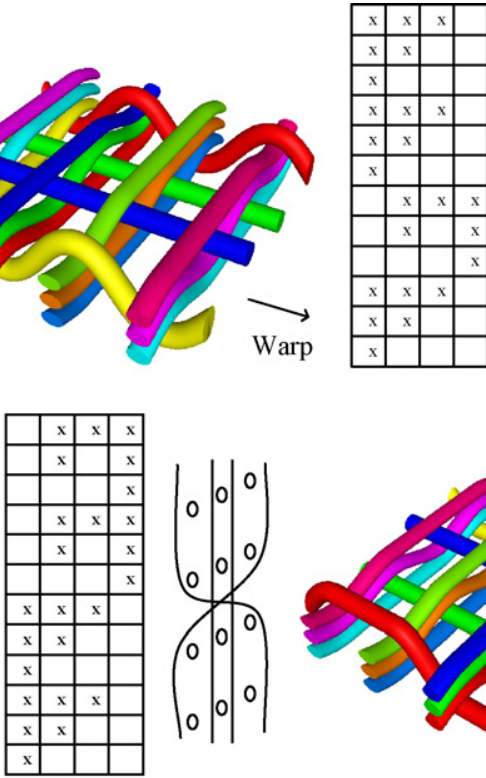

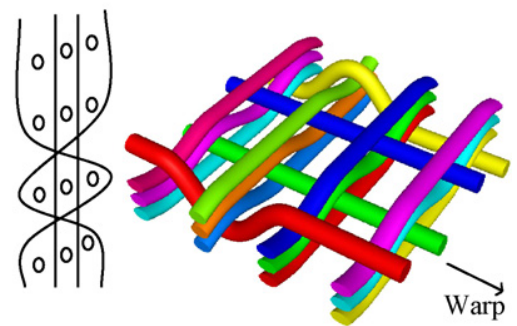

N3P2
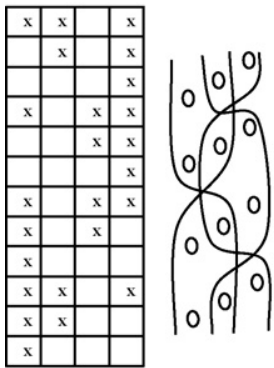

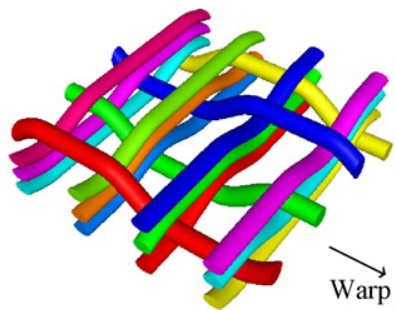

N3P4

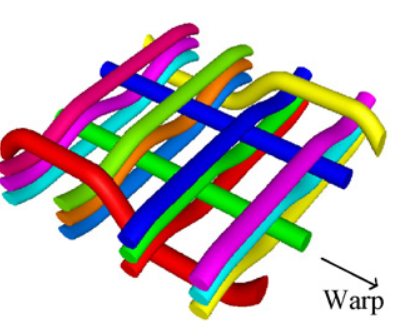

N3P3

Fig. 2. 3-Ply multilayer woven structures.

tenacity nylon-6 filament yarn (96 Tex). The graphical representation of the woven design and the line diagrams of 3-ply multilayer woven structures are represented in Fig. 2. Nylon 6 plain woven (half the weight, gsm, of multilayer fabric) is used as a reference sample for comparison to the above mentioned multilayer samples. The majority of the woven preforms used for composite reinforcement are plain woven, stacked one over another to achieve the desired fibre volume fraction. Hence two layers of the woven preform stacked one over the other are used as reinforcement and achieve the same volume fraction as control samples for comparison purposes.

Multilayer structures N3P1, N3P2 and N3P3, referred to as axial yarns (A), contain a relatively straighter set of warp yarns at the centre compared to regular interlacing warp yarns. The general construction characteristics of the fabric samples are provided in Table 1 , where $n_{1}$ and $n_{2}$ denote ends $/ \mathrm{cm}$, picks $/ \mathrm{cm}, c_{1}$ and $c_{2}$ represent warp and weft crimp percentage (\%) values and $I$ is the
Interlacement Index of the multilayer woven structure. Composites are prepared with these preforms by a hand-layup method using unsaturated polyester resin at ambient temperature and curing for one day with thickness spacer of $1.6 \mathrm{~mm}$.

The basic properties of prepared composites are presented in Table 2. Fibre volume fraction $\left(V_{\mathrm{f}}\right)$ of the composite was calculated using Eq. (2), with fibre density $\left(\sigma_{\mathrm{f}}\right)$, composite density $\left(\sigma_{\mathrm{c}}\right)$ data from density gradient tests along with weight $\left(\mathrm{g} / \mathrm{m}^{2}\right)$ of fabric $\left(W_{\mathrm{f}}\right)$ and composite $\left(W_{\mathrm{c}}\right)$. Void percentage $(\%)$ was estimated using an optical microscopy image analysis technique as per Purslow [20]. For this method, 50 images of each composite were captured using Leica microscope $(40 \times)$ fitted with digital camera. A representative image of the composites is displayed in Fig. 3. These images were analysed for presence of voids and average void percentage calculated from the ratio of void area to the total area of the composite. The tensile properties of the composites were evaluated as per ASTM D 3039 standard on an Instron universal tester. Flexural

Table 1

Multilayer woven interlocked preform properties (CV\% values are given within parenthesis).

\begin{tabular}{|c|c|c|c|c|c|c|c|}
\hline & \multirow[t]{2}{*}{$n_{1} \times n_{2}(\mathrm{~cm})$} & \multicolumn{2}{|l|}{ c1 (\%) } & \multirow[t]{2}{*}{ c2 (\%) } & \multirow[t]{2}{*}{$t(\mathrm{~mm})$} & \multirow[t]{2}{*}{ Weight $\left(\mathrm{g} / \mathrm{m}^{2}\right)$} & \multirow[t]{2}{*}{$I$} \\
\hline & & Regular & Axial & & & & \\
\hline N3P1 & $25 \times 12$ & $8.3(2.2)$ & $4.3(5.7)$ & $3.1(3.0)$ & 1.22 & $422.2(2.9)$ & 1.08 \\
\hline N3P2 & $24 \times 12$ & $5.6(1.9)$ & $3.1(2.9)$ & $2.1(4.1)$ & 1.34 & $420.1(3.2)$ & 0.96 \\
\hline N3P3 & $24 \times 12$ & $6.1(3.5)$ & $4.9(2.1)$ & $3(1.9)$ & 1.32 & $438.5(2.5)$ & 1.00 \\
\hline N3P4 & $24 \times 13$ & $6.5(3.9)$ & NA & $4(3.3)$ & 1.27 & $436.8(1.4)$ & 0.92 \\
\hline N3P5 & $24 \times 12$ & $6.8(4.8)$ & NA & $3.6(4.4)$ & 1.41 & $437.2(2.9)$ & 0.83 \\
\hline NP-control & $12 \times 6$ & $7.3(4.2)$ & NA & $4.6(2.3)$ & 0.71 & $212.3(4.5)$ & 2.00 \\
\hline
\end{tabular}


Table 2

Properties of multilayer reinforced composites.

\begin{tabular}{|c|c|c|c|c|c|c|c|}
\hline & \multirow[t]{2}{*}{$t(\mathrm{~mm})$} & \multirow[t]{2}{*}{$V_{\mathrm{f}}$} & \multirow[t]{2}{*}{ Void (\%) } & \multicolumn{2}{|c|}{ Tensile strength, (GPa) } & \multicolumn{2}{|c|}{ Flexural strength, (GPa) } \\
\hline & & & & Warp & Weft & Warp & Weft \\
\hline N3P1 & 1.65 & 0.28 & 2.97 & 116.21 & 66.39 & 39.62 & 31.02 \\
\hline N3P2 & 1.64 & 0.27 & 2.29 & 114.84 & 64.92 & 49.81 & 29.05 \\
\hline N3P3 & 1.64 & 0.28 & 1.84 & 110.91 & 66.68 & 50.14 & 31.71 \\
\hline N3P4 & 1.65 & 0.28 & 1.90 & 116.01 & 66.88 & 51.39 & 31.56 \\
\hline N3P5 & 1.63 & 0.32 & 2.55 & 117.19 & 71.78 & 51.09 & 30.04 \\
\hline NP-control & 1.62 & 0.31 & 3.91 & 108.3 & 63.1 & 50.1 & 30.6 \\
\hline
\end{tabular}

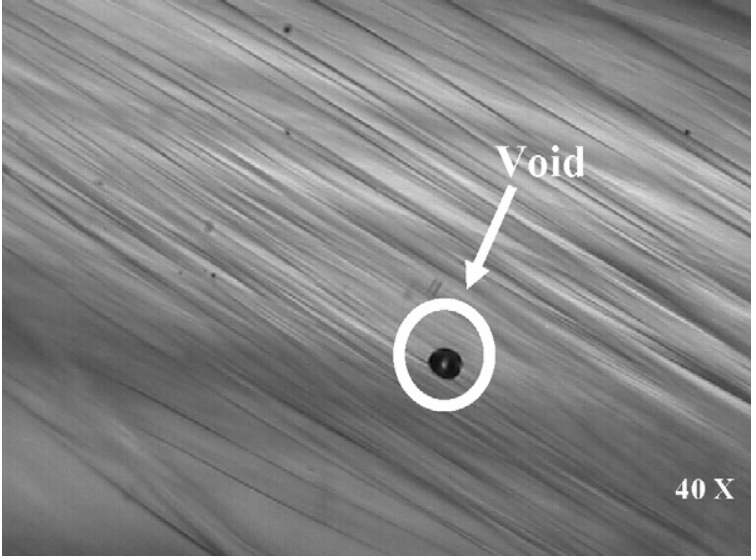

Fig. 3. Typical optical image of composites used for void estimation.

tests were carried out on the same Instron tester following ASTM Standard D 790.

$V_{\mathrm{f}}=\frac{W_{\mathrm{f}} / \sigma_{\mathrm{f}}}{W_{\mathrm{c}} / \sigma_{\mathrm{c}}}$

\subsection{Tribological experimental details}

The wear tests are conducted on a Universal Tribometer (UMT) with a reciprocating sliding system, supplied by CETR Inc., (Center for Tribology, USA). The schematic diagram of the experimental setup is shown in Fig. 4. The experimental set-up can accommodate test samples of various shapes and dimensions and can perform common tribological tests, such as ball/pin-on-disc or ball/pin-onplate.

The applied loading is servo-controlled (with a closed loop feed back) and can vary between a load cell range from $2 \mathrm{~N}$ to $200 \mathrm{~N}$. Load cell calibration was done at $20 \mathrm{~N}$ (the applied load in the described experiments). The test configuration was a ball-on-plate

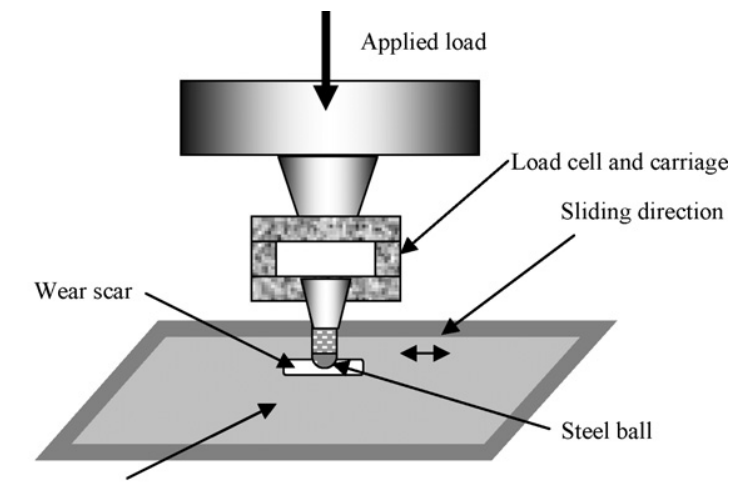

Textile composite sample (flat surface)

Fig. 4. Schematic diagram of the experimental set up for reciprocating sliding tests. system, where the steel ball (AISI 52100 ) was $10 \mathrm{~mm}$ in diameter and the polymer composite sample was mounted as a plate. The reciprocating sliding system has the amplitude of $4 \mathrm{~mm}$. All sample surfaces were cleaned using standard procedures. As the objective of this study is to evaluate the effect of frequencies on the tribological behaviour, a pilot study was conducted to optimize sliding distance and load. It was observed that the minimum required sliding distance is around $60 \mathrm{~m}$ in order to achieve a stabilized evolution of friction coefficient values and measurable wear scars for the selected frequency range $(0.5-8 \mathrm{~Hz})$.

An applied load of $20 \mathrm{~N}$ was selected based on a previous study of textile composites [10] and to avoid the high vibrations of the test system expected at high frequencies. The running time or sliding duration of the tests (corresponding to the sliding distance of $60 \mathrm{~m}$ ) were estimated for frequencies of $0.5 \mathrm{~Hz}, 1 \mathrm{~Hz}, 2 \mathrm{~Hz}, 4 \mathrm{~Hz}, 8 \mathrm{~Hz}$. The sliding tests were conducted on all six types of textile composite samples, starting with the control sample, and then the samples containing 3-ply woven interlocked structures as reinforcements (N3P1, N3P2, N3P3, N3P4 and N3P5). Finally, to understand the effect of load, one frequency is chosen $(4 \mathrm{~Hz})$ and tests were conducted under loads ranging from $5 \mathrm{~N}$ to $40 \mathrm{~N}$. All the tests were conducted at room temperature and repeated three times to estimate the error in the test, which was in the range of $\pm 5-7 \%$. Morphological characterization of the worn surfaces was done with an optical microscope and scanning electron microscopy. Wear volume estimation was done by measuring a dimension of the wear scar (width and length) and using an empirical mathematical equation that assumes the wear scar is formed by perfect ball geometry.

\section{Results and discussion}

\subsection{The effect of frequency and load on the tribological behaviour}

Fig. 5 shows the evolution of the average friction coefficient values as a function of sliding frequency. Composite N3P1 has the highest friction coefficient. It is clear that the presence of fibres in textile composites can result in very different friction coefficients. This could be due to the combined effect of voids in the matrix and

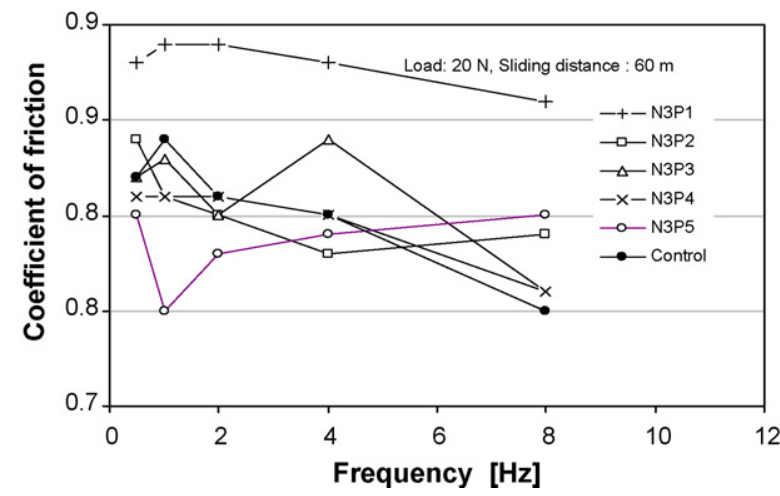

Fig. 5. Variation of friction coefficient values as a function of sliding frequency. 


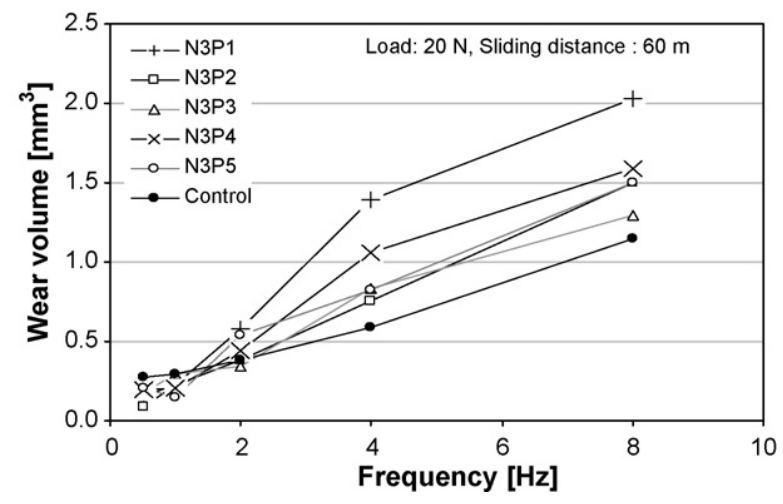

Fig. 6. Variation of wear volume as a function of sliding frequency.

different numbers of fibres oriented perpendicularly to the sliding wear direction. Fig. 5 also reveals a slight reduction in the friction coefficient values with increased frequency. Wang et al. [21] studied the effect of frequency on the friction behaviour of the polyethylene. They observed that the polyethylene friction coefficients increased with the frequency of sliding. However, at a certain load it could be reversed, as the action of load and frequency together could smooth the surface and reduce friction. Contact modes of the friction pairs also influence friction and temperature rise and its distribution in the contact region. Further, temperature is a key factor in determining the viscoelastic properties of the polymers and therefore has a great effect on the friction coefficient. Peter et al. [22] investigated the effect of ultrasonic fretting conditions on the tribological behaviour of friction materials. They suggested that at high frequency, friction-induced vibrations leads to friction forces much lower in magnitude than that predicted by quasi-stationary sliding friction. Hence, the reduction trend in friction coefficient as a function of frequency is explainable.
The variations of wear volume as a function of frequency are shown in Fig. 6. Wear volume increases with frequency, and below a frequency of $2 \mathrm{~Hz}$ the wear volume is very low. At frequencies larger than $2 \mathrm{~Hz}$ there is a large increase in the wear volume. Textile composite N3P1 has the highest wear volume at $2 \mathrm{~Hz}$ and greater frequencies, similar to the friction coefficient results (Fig. 5). The unique behaviour of N3P1 is addressed in a later Section 3.2. The wear volume ranking order of the other composites is N3P4 $>$ N3P5 $>$ N3P2 > N3P3 with N3P3 having the least wear volume.

In all tests, the AISI 52100 steel ball counterface was characterized by the absence of morphological changes, without any evidence of measurable wear.

Fig. 7 shows representative morphological features of N3P1 worn surfaces under different frequencies of the reciprocating sliding under an applied normal load of $20 \mathrm{~N}$. After testing at a low frequency $(0.5 \mathrm{~Hz})$, the worn surface of this composite reveals voids in the matrix and material removal without fibre exposure (Fig. 7(a)). The presence of voids is consistent with the fact that N3P1 has the highest void percentage (2.97\%, Table 2 ). The absence of exposed fibres is consistent with the low wear volume measured when frequencies are less than $2 \mathrm{~Hz}$ (Fig. 6). Another typical characteristic of lower frequency testing that is common to all textile composites was the presence of cracks in the matrix material, particularly around the fibre exposed regions (Fig. 7(b)). There is also evidence of cracks in the N3P1 composite matrix for a test at $2 \mathrm{~Hz}$, where the fibres are exposed by a large void in the matrix (Fig. 7(c)). Fig. 7(d) shows large fibre exposure at $8 \mathrm{~Hz}$ sliding frequency, corroborating the trend of increasing wear volume with increasing frequency (Fig. 6).

Fig. 8(a) and (b) presents the worn surfaces of N3P2 multilayer textile composite after reciprocating sliding at $4 \mathrm{~Hz}$, where removal of the matrix with some exposure of fibres can be observed (Fig. 8(a)). There is clear evidence of broken fibres occurring out of
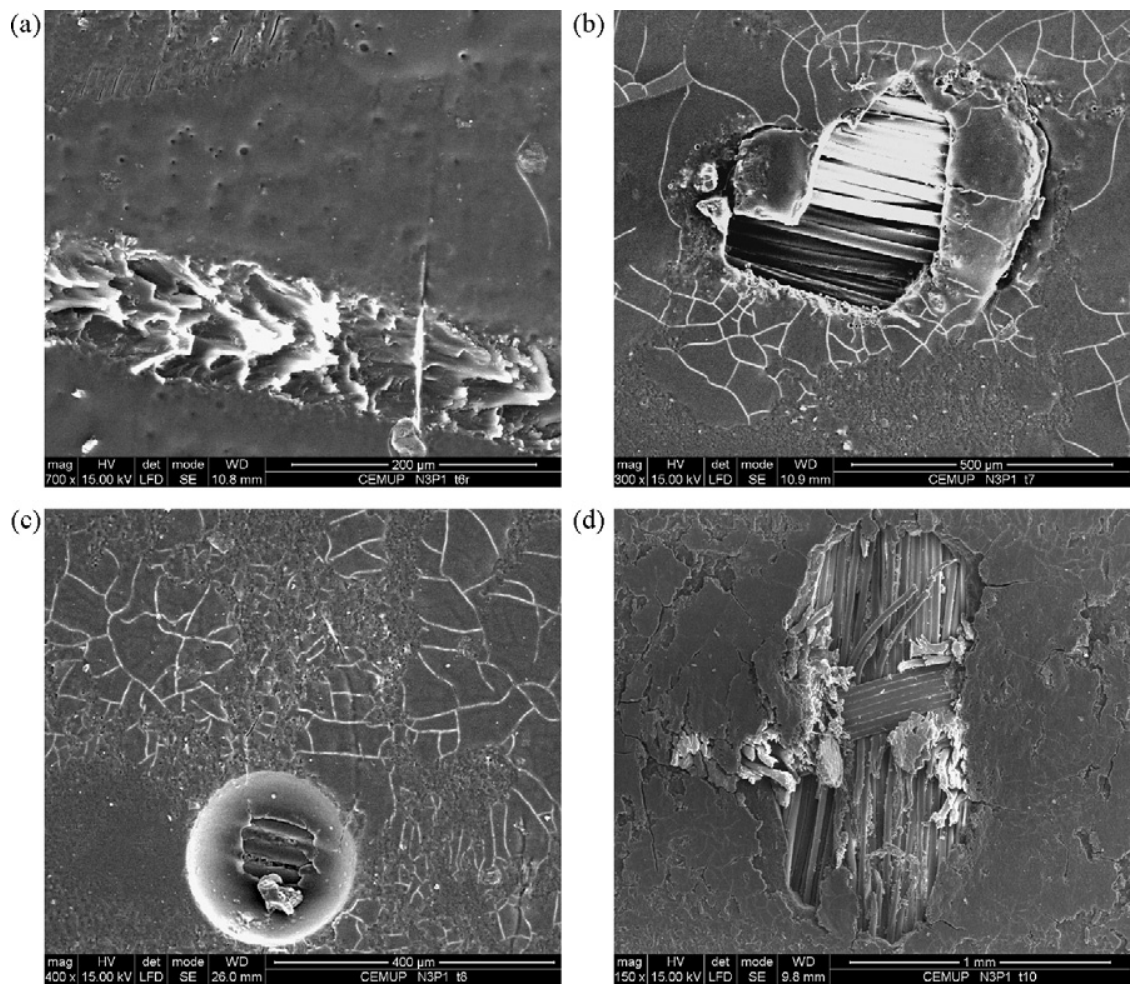

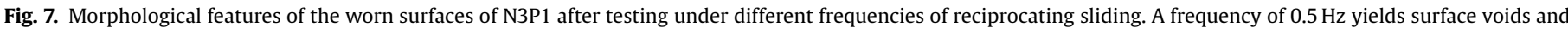

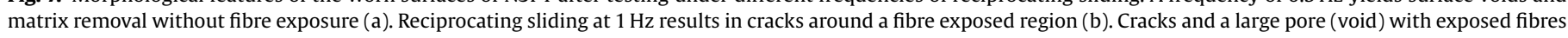
are visible for a frequency of $2 \mathrm{~Hz}$ (c). Large fibre exposure is visible when reciprocating sliding occurs at $8 \mathrm{~Hz}$ (d). 
(a)

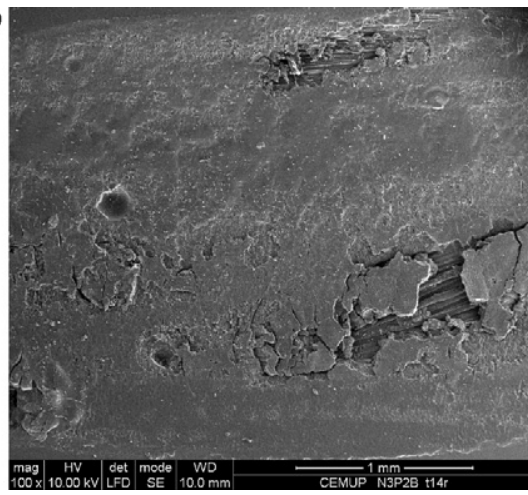

(b)

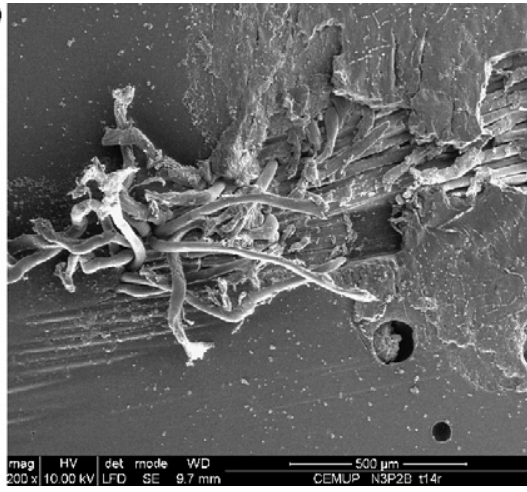

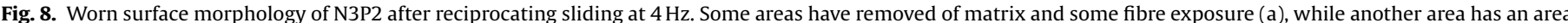
of fibres broken out of the boundary (b).
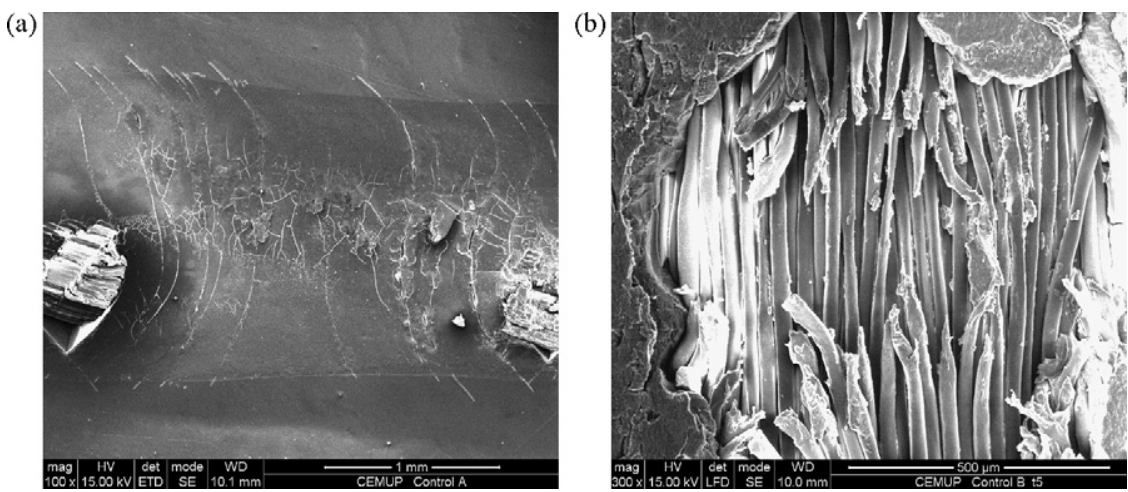

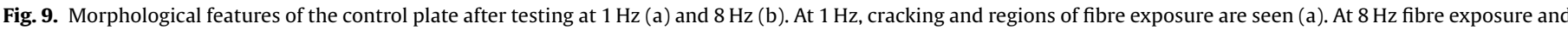
broken fibres are present (b).

the boundary (Fig. 8(b)). Contrary to the tests at low frequency, no extensive cracks are observed in the matrix material. The increased contact temperature at higher frequencies affects the viscoelastic behaviour of the matrix, reducing its brittle response. In fact, the increased contact temperature will also influence the sliding process, which depends on the glass transition temperature of the polymer matrix and properties of the fibre $[9,10]$.

Representative morphological features of the control plate are presented in Fig. 9. Again, the presence of cracks and regions of fibre exposure are very clear for a test at $1 \mathrm{~Hz}$, Fig. 9(a), whereas fibre exposure and broken fibres can be seen at an oscillating frequency of $8 \mathrm{~Hz}$ (Fig. 9(b)).

As previously mentioned, wear mechanisms of textile or polymer composites as a function of sliding frequency have been reported by several other researchers [7-10]. In the current study, the acting wear mechanism initiates matrix cracks in the composites at low wear frequency. Increasing sliding frequency results in ploughing of resin, followed by propagation of matrix cracks around the fibres. At the highest frequencies, matrix removal occurs which exposes the fibres resulting in fibre damage.

In addition, both normal and tangential loads are transmitted through the contact points during sliding by adhesive and ploughing actions. An increase in sliding frequency increases the rate of impact-type repeated loading on the surface. This increases the frictional thrust, which in turn causes localized vibration and chattering at the sliding interface; thereby increasing the debonding and fracture of the reinforcing fibres [6]. The broken bundles of fibres observed at high frequencies ( 4 and $8 \mathrm{~Hz}$, Figs. 8(b) and 9(b), respectively), is consistent with the high wear volume at these frequencies.

The overall effect of localized chattering and vibration in promoting debonding and breakage of reinforcing fibres in the plain weave woven fabric composite (control sample) is less than that of the other multilayer composites. Thus, the plain woven reinforced sample displayed better resistance to wear than other multilayer composites in the present study. The higher interlacement of weave for the plain weave woven fabric results in greater yarn locking, thus offering increased inter-yarn frictional resistance. The interyarn frictional resistance, in addition to the bonding effect of the matrix resin, provides extra strength to hold the yarns in position [6].

One interesting finding is that two different behaviours describe the relationship between wear volume and load at a constant frequency (Fig. 10) for the N3P2 composite. As the load increases from $5 \mathrm{~N}$ to $20 \mathrm{~N}$, there is a slow but steady increase in wear volume. However, as the load increases from $20 \mathrm{~N}$ to $40 \mathrm{~N}$ there is a sudden increase in wear volume. Therefore, the $20 \mathrm{~N}$ load used for the coefficient of friction studies is justifiable as it is the upper limit

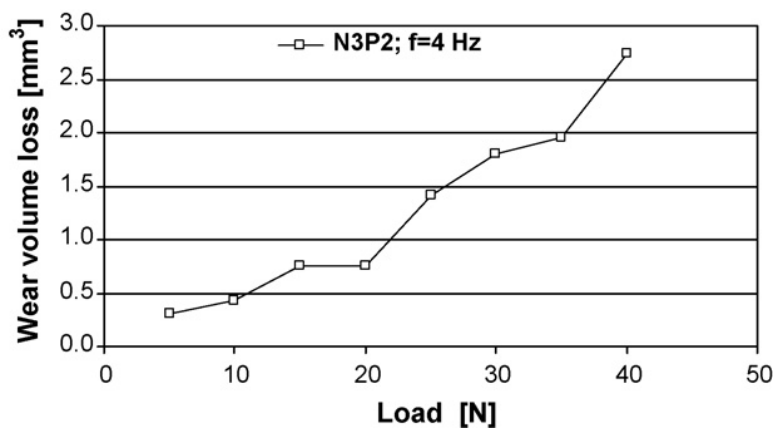

Fig. 10. Variation of wear volume as a function of applied load for the N3P2 composite. 


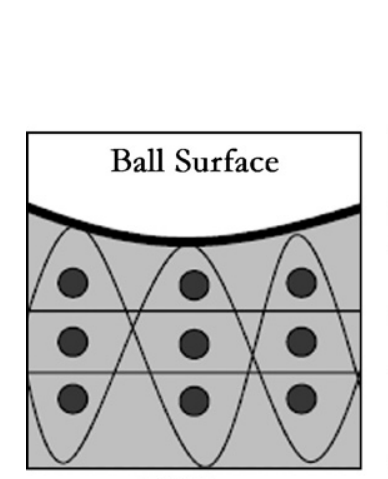

N3P1
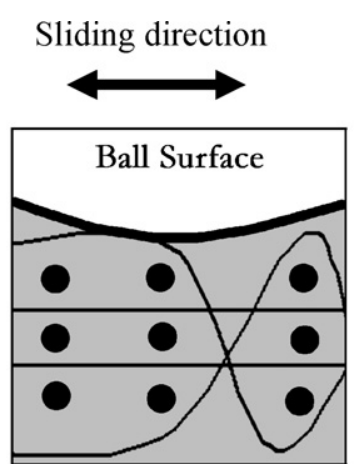

N3P2

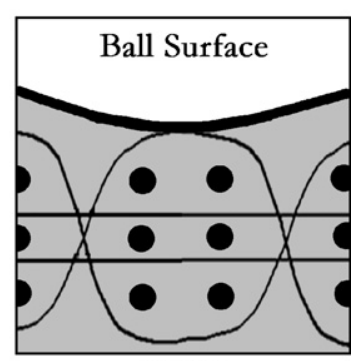

N3P3

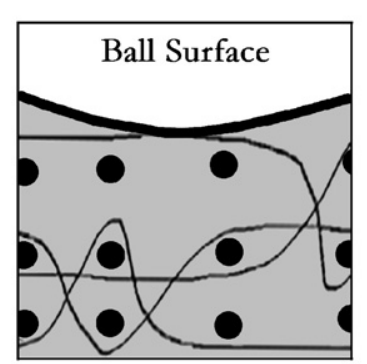

N3P4

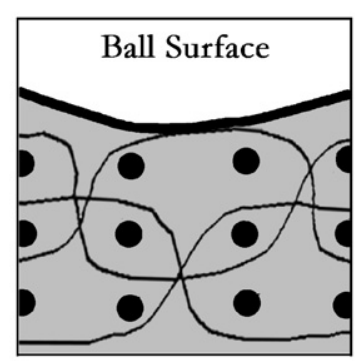

N3P5

Fig. 11. Schematic diagram of the contact zones for five different multilayer textile composites.

where steady state wear volume can be attained. It is clear that it is important to select the correct applied load for the specific tribological application and sliding frequency. More details on the effect of load and possible wear mechanisms on the tribological behaviour of textile composites are reported in a previous study [10].

\subsection{Tribological behaviour and Interlacement Index (I)}

A schematic diagram of the contact zone (between the ball and the textile composite) illustrates the differences in composite fibre arrangement for the five textile composites during sliding (Fig. 11). In the case of N3P1 the ball surface will articulate against more fibres aligned perpendicular to the sliding direction and articulate against more voids compared to the other four multilayer textile composites (Table 2). As a direct consequence, N3P1 is characterized by a high coefficient of friction and wear volume compared to the other textile composites studied (Figs. 5 and 6). For the N3P2, N3P3, N3P4, and N3P5 multilayer structures, the ball surface is in contact with reinforcement fibres at an angle closer to parallel with respect to the sliding direction (Fig. 11).

The effect of such structural fibre geometry in these multilayer fabrics (N3P2-5) can be evaluated using the Interlacement Index $(I)$. A higher $I$ means higher interlacement, hence more alignment of fibres perpendicular to the sliding direction. Fig. 12 portrays the relationship between Interlacement Index and the wear volume for the composites involved in the current study except N3P1. It can be seen that high index $(I)$ corresponds to high wear resistance of the five multilayer composites. The control sample (with the highest Interlacement Index, $I=2.0$ ) has the best wear resistance, thus the presence of high interlacement in the structure increases the resistance of the composite to fibre pull-out. In multilayered structures if the interlacement is higher, the number of fibres perpendicular to the sliding direction is higher, and correspondingly a higher wear resistance is observed. Both the N3P1 composite and the control sample have a high void content (Table 2 ). The voids in two layer stacked plain woven reinforced composites (the structure present in the control sample) are usually between the two layers and do not come immediately in contact with the ball during sliding. In con- trast, N3P1 has a multilayered interlocked structure that is used as a single layer for composite preparation and, therefore, the voids are usually near the surface of the composite next to the ball. A high number of voids near the composite surface would induce high wear volumes during sliding wear tests and correspond to the test results.

\subsection{Limitation and scope of the study}

It is worth in mentioning that the current study focused on one load with varying frequencies under a dry sliding condition. However, as it was mentioned, the combination of load and frequency has a significant effect on the tribological process. Hence, further study is required to consider additional tribological parameters and sliding conditions (e.g., lubricated conditions, or in presence of slurry). The textile composites were also manufactured with a hand-layup technique, and the manufacturing process can significantly influence the quality of the resulting laminate. This is evidenced by the higher presence of voids in all the samples, which should ideally be less than $1 \%$. Future studies will be planned with the manufacturing process taken into consideration. Studies will also be focused on developing a mathematical model to predict

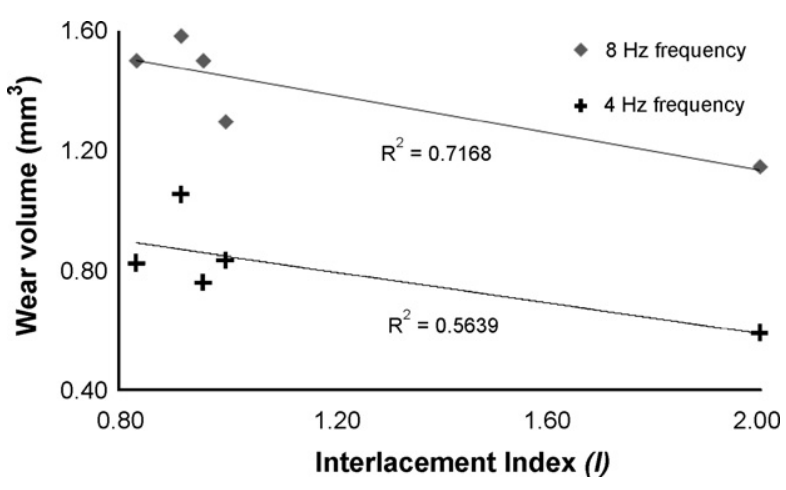

Fig. 12. Influence of the fibre reinforcement structure on wear behaviour. 
and/or verify the resulted wear volume loss of such complex textile composites $[7,8]$.

\section{Conclusions}

Five types of 3-ply multilayer interlocked woven structures with varying interlacements were prepared using nylon fibre and they were reinforced with polyester resin to prepare composite samples. These composite samples were subjected to reciprocating sliding wear to understand the influence of interlacement on the tribological behaviour of the composites in terms of wear volume and friction coefficient. Experimental investigation to understand the effect of sliding frequency on the tribological behaviour of such multilayered textile composites was conducted to explain the wear mechanisms involved in the tribological process. The following conclusions are drawn from the current study.

- The reinforcement structure has a strong influence on the tribological behaviour of textile composites with N3P1 presenting $90 \%$ more wear resistance than N3P3.

- Interlacement Index (I), representing structural geometry, can be used to explain the effect of yarn interlacements in multilayer structures on the tribological behaviour of textile composites. A higher Interlacement Index corresponds to a higher wear resistance of the material.

- The control sample with a plain woven reinforcement structure (highest interlacement) has the best wear resistance. N3P1 had the highest wear volume and friction coefficient values, attributed to the combined effect of higher void and perpendicular fibre orientation in the structure. The ranking order of materials from highest of lowest wear volume is N3P4 $>$ N3P5 $>$ N3P2 $>$ N3P3.

- At higher sliding frequencies (greater than $2 \mathrm{~Hz}$ ) the resulting wear volumes increased sharply compared to approximate steady state values at sliding frequencies less than $2 \mathrm{~Hz}$. Hence it is highly critical to select the load and frequency based on the specific application where such composites will be employed.

- Along with the structural geometry/fibre direction, matrix properties of the composite material also influence the acting wear mechanisms.

\section{Definition of the terms}

1. Woven fabric: A material constructed of interlaced yarns, fibres or filaments.

2. Composite material: Dissimilar materials laminated together to form a single structure. A material created from a fibre (reinforcement) and an appropriate matrix (polymeric resin) material in order to maximize specific performance properties. The constituents do not dissolve or merge completely but retain their identities as they act in concert.

3. Reinforcement: A material added to the matrix to provide the required properties; ranges from short fibres through complex textiles forms. A ready to use textile form to specific shape of composite mould is termed as 'Preform'.

4. Multilayer interlocked fabrics: Multilayer interlocked fabrics are a quite distinctive class of 3D textile structures manufactured by interlocking of fabric layers during the weaving stage. Multilayer interlocked fabrics are composed of several series of two sets of yarns (warp and weft) that form distinct layers and these layers are bound by interlacing warp yarns. 3-ply multilayer structures are 3 layers of warp and/or weft yarns interlocked together during weaving stage.

\section{Acknowledgments}

The authors would like to express their gratitude for financial support provided by FCT, Portugal, through an individual project (SFRH/BPD/20344/2004), and an Asia Link program between the University of Minho, Portugal, and IIT Delhi, India, sponsored by the European Union. Thanks also to Mr. Sérgio Carvalho, who assisted with experimental work in the Tribology Lab at University of Minho.

\section{References}

[1] D. Hull, T.W. Clyne, An Introduction to Composite Materials, Cambridge University Press, Cambridge, 1996.

[2] S. Nak-Ho, N.P. Suh, Effect of fibre orientation on friction and wear of fibre reinforced polymeric composites, Wear 53 (1979) 129-141.

[3] K.J. Lee, H.Z. Cheng, W.S. Jou, G.J. Chen, C.W. Liang, The influence of carbon fibre orientation on the mechanical and tribological behavior of carbon fibre/LCP composites, Materials Chemistry and Physics 102 (2007) 187-194.

[4] Qian-qian Shangguan, Xian-hua Cheng, Tribological properties of lanthanum treated carbon fibres reinforced PTFE composite under dry sliding condition, Wear 262 (2007) 1419-1425.

[5] M. Cirino, K. Friedrich, R.B. Pipes, The effect of fiber orientation on the abrasive wear behavior of polymer composite materials, Wear 121 (1988) 127-141.

[6] B. Vishwamath, A.P. Verma, C.V.S. Kameswara Rao, Effect of fabric geometry on friction and wear of glass fibre-reinforced composites, Wear 145 (1991) 315-327.

[7] K. Yoshioka, J.C. Seferis, Modeling of tensile fatigue damage in resin transfer molded woven carbon fabric composites, Composites: Part A 33 (2002) 1593-1601.

[8] K.Y. Tsang, D.L. DuQuesnay, P.J. Bates, Fatigue properties of vibration-welded nylon 6 and nylon 66 reinforced with glass fibre, Composites: Part B 39 (2008) 396-404.

[9] Shirong Gea, Shibo Wang, Norm Gitis, Michael Vinogradov, Jun Xiao, Wear behavior and wear debris distribution of UHMWPE against $\mathrm{Si}_{3} \mathrm{~N}_{4}$ ball in bidirectional sliding, Wear 264 (2008) 571-578.

[10] M.T. Mathew, V. Naveen, L.A. Padaki, J.R. Rocha, R. Gomes, B.L. Alagirusamy, R. Deopura, Fangueiro, Tribological properties of the directionally oriented warp knit GFRP composites, Wear 263 (2007) 930938.

[11] N.K. Myshkin, M.I. Petrokovets, A.V. Kovalev, Tribology of polymers: adhesion, friction, wear, and mass-transfer, Tribology International 38 (2005) 910-921.

[12] K Friedrich, R. Reinicke, Z. Zhang, Wear of polymer composites, in: Proceedings of the Institution of Mechanical Engineers, Part J: Journal of Engineering Tribology 216 (6) (2002) 415-426.

[13] Surya D. Pandita, Get Huysmans, Martine Wevers, Ignaas Verpoest, Tensile fatigue behaviour of glass plain-weave fabric composites in on-and off-axis directions, Composites: Part A 32 (2002) 1533-1539.

[14] M.O.W. Richardson, M.J. Wisheart, Review of low-velocity impact properties of composite materials, Composites: Part A 27A (1996) 1123-1131.

[15] C. Sabit Adanur, Andrew Tam, On-machine interlocking of 3D laminate structures for composites, Composites Part B 28B (1997) 497-506.

[16] Huang Hu, Comparison between laminated and integrated glass fibre reinforced plastics, Materials \& Design 21 (2000) 461-464.

[17] Huang Hu, Zhilli Zhong, Tensile behaviour of 3D woven composites by using different fabric structures, Materials \& Design 23 (2002) 671-674.

[18] V. Milasius, R. Milasius, E. Kumpikaite, A. Olsauskiene, Influence of fabric structure on some technological and end- use properties, Fibres \& Textiles in Eastern Europe 11 (2) (2003) 41-47.

[19] N.V. Padaki, B.L. Deopura, R. Alagirusamy, B.S. Sugun, R. Fangueiro, Influence of preform interlacement on the low velocity impact behaviour of multilayer textile composites, in Proceedings of International conference on technical textiles and non wovens (ICTN 2008), New Delhi, 2008, 4B-5.

[20] D. Purslow, On the optical assessment of the void content in composite materials, Composites 15 (1984) 207-210.

[21] Qi. Wang, K. Xiagan, Z. Lushan, Ji Zhe, FYing, Study on the tribological behaviour of polyethelene. II. Effects of operating conditions on the friction behaviour of polyetehelene, Journal of Applied Polymer Science 58 (1995) 903-910.

[22] Peter Rehbein, Jörg Wallaschek, Detlef Löhe, A test method to investigate the tribological behaviour of friction materials under ultrasonic fretting conditions, Tribotest 6 (2006) 1-16. 